\title{
Mapeo del campo de conocimiento en intenciones emprendedoras mediante el análisis de redes sociales de conocimiento
}

\author{
Field mapping of knowledge in entrepreneurial intentions \\ through analysis of social knowledge network \\ Iván Montoya Restrepo $^{1} \quad$ Alejandro Valencia Arias $^{2} \quad$ Alexandra Montoya Restrepo $^{1}$ \\ Recibido 12 de marzo de 2015, aceptado 10 de septiembre de 2015 \\ Received: March 12, $2015 \quad$ Accepted: September 10, 2015
}

\begin{abstract}
RESUMEN
Este artículo tiene como objetivo examinar la estructura del conocimiento en el campo de las intenciones emprendedoras mediante el análisis de redes sociales de conocimiento (autores y de temas de investigación), para identificar posibilidades de sinergias, interacciones y nuevos estudios en dicho campo de conocimiento. La metodología utilizada se basa en una revisión sistemática de literatura en Scopus, cuya ecuación de búsqueda arrojó como resultado 202 artículos, los cuales se analizan de forma visual y cuantitativa, involucrando redes de coautoría y de coocurrencia de palabras claves, con el fin de obtener la visualización de redes de autores y redes de temas de investigación en niveles macro y micro, respectivamente. La construcción de la estructura de red y cálculo de la red central se llevaron a cabo mediante el modelo de grafo binario no dirigido y el Keyword Co-occurrence Network, mediante los software libre: Bibexcel, Netdraw y Gephi. Entre los resultados se observa que el modelo más utilizado en este campo de estudio es la Teoría del Comportamiento Planeado, siendo el referente conceptual sobre el cual se han construido la mayoría de nuevos modelos. Además, el Modelo de Ecuaciones Estructurales se ha consolidado como el camino más utilizado para el análisis e interpretación de la información primaria recolectada. Frente a esto, se plantea la necesidad de generar nuevos campos de estudio en el área de las intenciones emprendedoras, por lo que se sugiere la incorporación de visiones no lineales apoyadas en herramientas sistémicas que brinden un mejor acercamiento al entendimiento del dinamismo y las relaciones de causalidad presentes en las variables de los modelos de intención emprendedora.
\end{abstract}

Palabras clave: Modelos de intención emprendedora, bibliometría, creación de empresas, redes sociales de conocimiento.

\section{ABSTRACT}

This article aims to examine the structure of knowledge in the field of entrepreneurial intentions through social knowledge network analysis (authors and research topics), which allows identifying potential synergies, interactions and new studies in this field of knowledge. The methodology is based on a systematic review of literature in Scopus with an equation of search that recovered 202 articles. The articles are analyzed visually and quantitatively, involving networks of co-authorship and co-occurrence of keywords to obtain visualization of networks of authors and research topics in macro and micro levels, respectively. The construction of the network structure and calculation of the core network is carried out through the model of binary undirected graph and KCO Network with free software: Bibexcel, Netdraw and Gephi. The results show that the model most commonly used in this field of study is the Theory of Planned Behavior. It is the conceptual reference on which have been built most of new models. In

1 Departamento de Ingeniería de la Organización. Universidad Nacional de Colombia. Dirección postal: Carrera 80 № $65-223$ Núcleo Robledo. Medellín, Colombia. E-mail: iamontoyar@unal.edu.co; lamontoyar@unal.edu.co

2 Departamento de Ciencias Administrativas. Instituto Tecnológico Metropolitano. Doctorando en Ingeniería-Industria y Organizaciones. Universidad Nacional de Colombia. Cra. 315422 Boston - Campus Fraternidad. Medellín, Colombia. E-mail: jhoanyvalencia@itm.edu.co 
addition, the Structural Equation Model has been consolidated as the most used method for the analysis and interpretation of collected primary data. Then, the need arises to generate new fields of study in the area of entrepreneurial intentions. Thus, it is raised the need to generate new fields of study in the area of entrepreneurial intentions. Therefore, it is suggested the incorporation of nonlinear visions supported by systematic tools that provide a better approach to understanding the dynamics and the causal relationships presented in the variables of entrepreneurial intention models.

Keywords: Models of entrepreneurial intention, bibliometrics, entrepreneurship, social knowledge network.

\section{INTRODUCCIÓN}

El estudio de la dinámica de la ciencia y la tecnología proporciona las claves necesarias para entender cómo se crean, crecen y cambian los diferentes campos del conocimiento. Además, los investigadores se enfrentan ante cambios en la demanda de ciencia y tecnología, haciendo necesario estudiar su evolución con el fin de proporcionar claridad a las partes interesadas acerca de los avances en los diferentes campos del conocimiento, y ayudar en la toma de decisiones y en la formulación de nuevos proyectos de investigación acordes con las necesidades del área de estudio [1].

Es por esto que existen varias razones que conducen a un mayor interés en el mapeo de información, entre las que se encuentran: la citación de datos, el estudio de la estructura y las tendencias en la literatura de varias disciplinas; una mayor disponibilidad, accesibilidad de datos y citas en servicios de indexación; y la disponibilidad de aplicaciones informáticas para visualización y mapeo [2]. Por otro lado, la comunidad científica estructura sus relaciones conforme a modelos de redes sociales de conocimiento, en las cuales, los actores (nodos) representan individuos, instituciones, disciplinas científicas, entre otros, y los enlaces, representan el conocimiento que intercambian dichos nodos.

Frente a esto, el análisis de redes sociales de conocimiento se posiciona como una herramienta que permite comprender la estructura de un campo investigativo específico al mostrar las relaciones entre los actores que lo componen [3]. En este artículo se examina el campo de estudio de las intenciones emprendedoras bajo metodologías de análisis de redes sociales de conocimiento mediante mapeo, mostrando los temas más importantes en estudio; así como los principales actores, sus relaciones en la investigación de esta temática y cómo estos se relacionan entre ellos. Con esto se busca identificar posibilidades de sinergias, interacciones y nuevos estudios en dicho campo de conocimiento. Todo lo anterior bajo la consideración que el estudio de las intenciones emprendedoras es de vital importancia porque las intenciones son consideradas como el mejor predictor de las conductas planeadas (comportamiento racional), entre ellas la motivación por emprender [4-5]. Para alcanzar el objetivo propuesto, el campo de estudio de la aplicación de las redes sociales de conocimiento en las intenciones emprendedoras se estudia por medio de un diseño metodológico cuantitativo al combinar teoría de redes, redes de colaboración y coocurrencia de palabras.

Inicialmente se plantea la pertinencia del análisis de redes sociales como alternativa para mapear el conocimiento desde la perspectiva del análisis bibliométrico, las redes de colaboración y las redes de coautoría. A partir de esta fundamentación se plantea el diseño metodológico desde el que se detalla el proceso de construcción de la estructura de red y el cálculo de la red central. Finalmente, el análisis de resultados plantea la construcción de mapas del conocimiento de intenciones emprendedoras, reportando las principales subáreas de estudio en este campo de conocimiento y el panorama futuro de la investigación en dicha temática.

\section{ANÁLISIS DE REDES SOCIALES COMO ALTERNATIVA PARA MAPEAR EL CONOCIMIENTO}

Una red social puede definirse como un conjunto de puntos (actores sociales) vinculados por relaciones que cumplen determinadas propiedades. Las redes sociales poseen una estructura y una morfología propias, cuyas cualidades, como la posibilidad de cuantificar las relaciones y su consiguiente tratamiento matemático, evidencian diversas 
aplicaciones para el análisis e interpretación de las conductas sociales [6].

El Análisis de Redes Sociales ha ganado importancia en las últimas dos décadas, debido a que plantea un nuevo paradigma que se centra en las características relacionales de los fenómenos sociales y patrones de comportamiento. Lo anterior le ha permitido diferenciarse de los enfoques científicos tradicionales que suelen analizar metodológicamente los diferentes objetos de investigación como independientes los unos de los otros [7-8].

Una red social se compone de diferentes tipos de actores sociales y se forma sobre la base del intercambio social, permitiendo responder las preguntas: ¿cómo se intercambian los recursos?, ¿cómo los actores sociales influyen en el intercambio de recursos? y ¿cuáles son los recursos más importantes? [9]. El análisis de redes sociales se basa en la premisa que las relaciones entre los actores pueden ser descritas por grafos, un conjunto de puntos (nodos) y sus uniones (vértices, aristas). Los nodos del grafo representan los actores sociales y las aristas representan las interacciones entre los actores. Esta representación permite a los investigadores aplicar la teoría de grafos, ya que de otra forma sería difícil la comprensión de las relaciones sociales $[8,10]$.

La perspectiva de las redes sociales involucra teorías, modelos y aplicaciones que son expresadas en términos de conceptos o procesos relacionales. Estas relaciones, definidas por vínculos entre unidades, son el núcleo central de la teoría de redes; pero en adición a esto, también son destacables los siguientes elementos [11]:

a. Los actores y sus acciones son considerados interdependientes.

b. Los vínculos entre actores son canales para transferir flujos de recursos.

c. Los modelos de red conceptualizan la estructura social, económica y política, como patrones duraderos de relaciones entre actores.

d. Los modelos de red se enfocan sobre los individuos, viendo el ambiente estructural de la red como proveedor de oportunidades para restringir acciones individuales.

Los científicos de una gran variedad de campos han desarrollado un amplio conjunto de herramientas matemáticas, computacionales y estadísticas para modelar, analizar y comprender las redes [12]. Esto ha ayudado a consolidar este enfoque como útil para el estudio de la interconexión entre los actores individuales o colectivos en los procesos sociales, como flujos de comunicación o situaciones de toma de decisiones [7]. De lo anterior se destaca que la ciencia de las redes sociales no solo se distingue por su materia objeto, sino además por su metodología y las características fundamentales de este enfoque que ayudan a comprender los sistemas complejos, consolidándose como un aporte en los siguientes aspectos [13]:

- Interdisciplinariedad: ofreciendo un lenguaje con el que las diferentes disciplinas pueden interactuar unas con otras, llevando a una fertilización transversal de herramientas e ideas.

- Empirismo: a pesar de tener sus raíces en la teoría de grafos, lo que distingue la ciencia de las redes sociales de conocimiento es su carácter empírico, es decir, su enfoque en los datos y en su utilidad.

- Naturaleza cuantitativa y matemática: uso del formalismo de los métodos matemáticos para hacer frente a la aleatoriedad y buscar principios organizativos universales.

- Naturaleza computacional: varias herramientas de software ayudan a los profesionales con habilidades computacionales a analizar las redes, debido al gran tamaño de las mismas.

\section{El análisis bibliométrico como herramienta para} hacer seguimiento a la evolución del conocimiento La bibliometría es el estudio cuantitativo de la literatura y la producción académica que recoge un conjunto de métodos matemáticos y estadísticos. Estos se usan para analizar y medir la cantidad y calidad de libros, artículos y otras formas de publicaciones, cuyos resultados sirven para la toma de decisiones frente a los enfoques de las nuevas propuestas investigativas [14-15].

Muchos campos de investigación utilizan métodos bibliométricos para explorar el impacto de su campo, el impacto de un conjunto de investigadores, o el impacto de un trabajo en particular [14]. El análisis de citas y otros métodos bibliométricos y cienciométricos comenzó a aplicarse de forma rudimentaria a pocos campos científicos. Hoy se aplican al estudio de las ciencias naturales y 
ciencias sociales y permiten su utilización en la formulación de políticas científicas de las instituciones de investigación, los gobiernos y otros organismos de financiación [2]. Sin embargo, el análisis bibliométrico se encuentra limitado por la información disponible, lo que hace necesario contar con fuentes de información confiables y adecuadas para realizarlo de manera eficiente y lograr una toma de decisiones más acertadas [16].

Mediante el enfoque bibliométrico es posible investigar la representación del conocimiento en el ámbito de la especialidad científica, en las estructuras intelectuales, en las redes formales e informales, tanto naturales como en ciencias sociales [1]. Esto hace posible encontrar patrones no evidentes, útiles para el avance de las investigaciones y el desarrollo científico [15], e incluso, para entender el pasado, la evolución del conocimiento, su estructura y potencialmente entender las tendencias futuras [17].

En bibliometría hay dos procedimientos principales: análisis de desempeño y la cartografía de la ciencia. El análisis del desempeño tiene como objetivo evaluar los grupos de actores científicos (países, universidades, departamentos, investigadores) y el impacto de su actividad sobre la base de los datos bibliográficos. Y la cartografía de la ciencia tiene como objetivo mostrar los aspectos estructurales y las dinámicas de la investigación científica [14].

Para facilitar el análisis se definen los indicadores bibliométricos como datos numéricos extraídos de los documentos que publican los investigadores. Estos indicadores pueden ser unidimensionales, es decir, estudian una sola característica de los documentos sin tener en cuenta ningún vínculo común entre ellos; y multidimensionales, los que permiten considerar simultáneamente las diversas variables o múltiples interrelaciones que se pueden extraer de documentos y hábitos de investigación de los autores [18].

Entre los indicadores más usados en los análisis bibliométricos se incluyen las revistas, las áreas de investigación e instituciones con mayor cantidad de publicaciones, los keywords (o palabras clave) más frecuentes, los autores más citados y las citaciones y cocitaciones de autores y revistas. Además, con base en el propósito del indicador, se han identificado tres tipos de indicadores bibliométricos: de cantidad (miden la productividad de un investigador), de calidad (miden el impacto de los resultados de los investigadores) y estructurales (miden las conexiones entre publicaciones, autores y campos del conocimiento). Al considerar la unidad de análisis se pueden identificar tres niveles de agregación: micro (autor), macro (región o país) y meso (la institución) [15].

Por otro lado, el análisis bibliométrico, con el objetivo de mapear la dinámica de la ciencia, normalmente utiliza mapas que buscan explorar la estructura subyacente de similitudes e interrelaciones entre los elementos o entidades. Entre ellos se destacan [1]:

- Mapas de copalabras: se basan en la identificación de pares de palabras claves, que ocurren juntas en un gran número de documentos. El marco supone que cuando dos palabras aparecen juntas en títulos o resúmenes, o en el texto completo de un mismo documento, se indica un posible vínculo entre dos centros distintos de interés designados por estas palabras. Cuando estas palabras se repiten en gran número de documentos pueden definir un subtema de investigación o subdominio intelectual que no ha entrado en la corriente principal de la delimitación de la investigación de ese campo.

- Mapas de coclasificación: se basan en el análisis de las coocurrencias de términos de clasificación subjetivos. Los términos de la clasificación, en su mayoría, representan elementos cognitivos, referidos a temas científicos, especialidades o campos.

\section{Redes de colaboración}

La informatización de las publicaciones científicas ha permitido la posibilidad de cuantificar y analizar los patrones que caracterizan la colaboración científica. Para alcanzar lo anterior se han presentado variados esfuerzos, entre los que se destacan la identificación de las citas entre los artículos (redes de citación) y la cartografía y el estudio de la colaboración entre los investigadores (redes de coautoría). Sin embargo, la coautoría implica un vínculo social mucho más sólido que la citación; ya que las citas pueden ocurrir sin que los autores se conozcan entre sí y 
pueden extenderse a lo largo del tiempo. Mientras que la coautoría implica una relación temporal y colegial que lo sitúa más de lleno en el campo de análisis de redes sociales de conocimiento [8, 19].

En las redes de colaboración los individuos se vinculan si se encuentran involucrados en una actividad común [20], facilitando el intercambio y la realización de tareas respecto de una meta compartida [21]. El análisis de redes de colaboración científica ha ejercido gran influencia en el desarrollo de la ciencia moderna de las redes. Este concepto se asocia con el término coautoría: dos científicos están vinculados si han sido coautores de al menos un artículo [20], y con base en esto cada investigador se asigna como un nodo, mientras que las autorías conjuntas establecen los vínculos entre los nodos [19].

Por lo anterior, en los últimos años se ha producido un marcado incremento en las colaboraciones entre científicos, ya que mediante la publicación de un documento conjunto se muestran actividades de intercambio de saberes, condición esencial para la creación de conocimiento [22]. Además, debido a la necesidad de mantener el ritmo de avances científicos, la mayoría de los gobiernos trazan políticas orientadas a mejorar el nivel de colaboración internacional. También, la colaboración científica facilita el aumento de la visibilidad de los investigadores altamente productivos, la creación de nuevos conocimientos científicos y la mejora en la calidad de la investigación [23].

Otra consideración importante que surge de las redes de colaboración es acerca del estudio de las comunidades, en las que se encuentran grupos de personas con intereses comunes de investigación en diferentes temáticas y disciplinas. Girvan y Newman [24] estudiaron la red de colaboración de los científicos que trabajan en el instituto Santa Fe, discriminando entre divisiones de investigación, tanto por tema como por metodología de la investigación. Los resultados mostraron cómo en las comunidades construidas en torno a temas de investigación, los científicos colaboran principalmente con otros, con los que su enfoque de investigación está estrechamente alineado. La formación de las comunidades en torno a las metodologías es más interesante y puede ser la marca de un verdadero trabajo interdisciplinario [24].

\section{Propiedades de las redes sociales de conocimiento aplicadas a redes de coautoría}

Para modelar una red de cocoautoría existen tres enfoques. El primer modelo es el tradicional grafo binario no dirigido (cada vínculo representa una relación de coautoría); el segundo modelo es una red dirigida binaria que permite el cálculo del prestigio del actor; $y$ en el tercer modelo se consideran las ponderaciones de las relaciones de coautoría en la red. En el caso de la red unidireccional, existen varios indicadores sociales para la medición de las diferentes propiedades de la red y algunos solo pueden aplicarse bajo ciertas condiciones [8]:

- Centralidad de grado: se define como el número total de vínculos (grado) de un autor $i$, considerando su estructura local (ego) [23], [9]. Esta medida representa la instancia más simple de la noción de centralidad, ya que solo mide el número de conexiones que unen los autores a sus vecinos más cercanos en la red. Sin embargo, los autores pueden estar bien conectados con sus vecinos inmediatos, pero ser parte de un grupo relativamente aislado, siendo así, la centralidad global baja [8]. De esta forma, esta medida es un indicador de la actividad de comunicación de un autor [23]. Lo anterior se representa con la ecuación (1).

$$
d(i)=\sum_{j} m_{j i}
$$

Donde $\mathrm{m}_{\mathrm{ij}}=1$, si el actor $i$ y el actor $j$ están vinculados.

- Centralidad de cercanía: mide lo cerca que un autor se encuentra a los demás autores. Un autor central se caracteriza por tener muchas conexiones cortas a otros autores en las redes [8]. El cálculo de esta medición sobre un actor, se define por la inversa de la longitud media de las rutas más cortas desde todos los otros actores de la red. Una mayor centralidad de cercanía indica mayor influencia sobre otros actores [9]. Esto se representa con la ecuación (2).

$$
c(i)=\sum_{i j}^{N} \frac{1}{d_{j i}}
$$

Donde $\mathrm{d}_{\mathrm{ij}}$ es el camino más corto entre el actor $j$ y el actor $i$. 
- Centralidad de intermediación: se basa en la determinación de la frecuencia en que un nodo particular es encontrado en el camino más corto (geodésica) entre cualquier par de nodos en la red. Los nodos que están a menudo en la ruta más corta entre otros nodos se consideran altamente centrales, debido a que controlan el flujo de información en la red (potencial de un actor a controlar la comunicación). Esos actores ubicados en el camino más corto entre otros actores están jugando un papel de intermediario que ayudan a dos actores sin contacto directo. Por otro lado, esta medida puede ser utilizada en redes desconectadas, aunque puede generar un gran número de nodos con centralidad cero, ya que muchos nodos pueden no actuar como un puente en la red [8-9, 23]. Esto se representa con la ecuación (3).

$$
b(i)=\sum_{j . k \neq 1} \frac{g_{j i k}}{g_{j k}}
$$

Donde $\mathrm{g}_{\mathrm{ik}}$ es el camino más corto entre el actor $j$ y el actor $k, \mathrm{~g}_{\mathrm{jik}}$ es el camino más corto entre el actor $j$ y el actor $k$ que contiene al actor $i$.

\section{DISEÑO METODOLÓGICO}

\section{Etapa 1. Revisión de literatura}

Las bases de datos actualmente son reconocidas como gestoras para la evaluación del quehacer investigativo de la comunidad científica mediante la aplicación de técnicas para construir indicadores bibliométricos [26].

La base de datos SCOPUS se utilizó en el proceso de obtención de documentos para extraer las palabras claves. Lo anterior, debido a sus ventajas tales como facilidad de navegación, indexación (100\% de los documentos) [27], multidisciplinariedad, amplitud del contenido [28] y flexibilidad de los operadores de búsqueda [29]. La estrategia de búsqueda utilizó las palabras "Entrepreneurial intention" y "Entrepreneurial intentions" considerando los campos de título y abstract, observando como unidad de análisis las publicaciones científicas, ya que estas proporcionan acceso a la dinámica de ciencia y contienen información respecto a las actividades científicas producidas bajo condiciones sociales [1]. Por lo anterior, se restringió la búsqueda a publicaciones tipo Article or review entre 1993 y
2013, buscando capturar la evolución del área de "intenciones emprendedoras" en el tiempo.

La ecuación de búsqueda contó con la siguiente estructura: (TITLE (entrepreneurial intention) OR TITLE (entrepreneurial intentions) OR ABS (entrepreneurial intention) ORABS (entrepreneurial intentions)) AND DOCTYPE (ar OR re) AND $P U B Y E A R>1992$. La información se recuperó de la base de datos el 7 de noviembre de 2014, arrojando 202 documentos, de estos 192 son artículos y 10 artículos de revisión; 195 documentos son en inglés, 9 en español, 3 en chino, 1 en francés, 1 en ruso y 1 en ucraniano. Además 26 documentos no presentaron palabras claves de autor.

\section{Etapa 2. Estandarización de palabras clave}

Algunas palabras se pueden utilizar de la misma manera para describir conceptos similares, por lo que algunas de ellas fueron estandarizadas para evitar entrar en conflictos semánticos o duplicidades conceptuales. La revisión, documento a documento, se hizo enfocada en el contexto de utilización de los conceptos, para identificar cuándo se podía agrupar bajo un concepto unificador, además de agrupar sinónimos y siglas. Por ejemplo, entrepreneurial intention y entrepreneurial intentions fueron agrupadas, por ser utilizadas para realizar los mismos tipos de análisis, en 2 diferentes papers. Del proceso anterior surgieron 873 palabras claves. El listado con las 23 palabras que presentaron frecuencia mayor que 1 se presenta a continuación (Tabla 1).

Tabla 1. Palabras clave con mayor frecuencia.

\begin{tabular}{|c|c|}
\hline Palabra clave & Frecuencia \\
\hline Entrepreneurial intentions & 98 \\
\hline Entrepreneurship & 54 \\
\hline Students & 41 \\
\hline Entrepreneurialism & 30 \\
\hline Theory of Planned Behavior (TPB) & 25 \\
\hline Education & 24 \\
\hline Entrepreneurship education & 20 \\
\hline Gender & 18 \\
\hline Self-efficacy & 17 \\
\hline Attitudes & 9 \\
\hline
\end{tabular}

Etapa 3. Construcción de la estructura de red y cálculo de la red central

La red de las palabras clave se basa en correlaciones. Una correlación se presenta como un "lazo de la red" [17]. Para generar los vínculos de la red se proponen dos métodos: 
- Modelo de grafo binario no dirigido: es un modelo sencillo y ampliamente utilizado, donde cada vínculo representa una relación de coautoría [8]. Este modelo fue utilizado para la construcción de la red de autores y redes de centros de investigación con base en un análisis desde el macro nivel [17].

- KCO Network (Keyword Co-occurrence Network): la relación entre las palabras clave se debe a que estas se indican en el mismo artículo. En este caso, las palabras claves encontradas en los artículos se vinculan porque todas estas pueden utilizarse para representar las ideas centrales de un trabajo de investigación. El análisis es realizado desde el micronivel con el objetivo de comprender la coocurrencia de palabras clave y las redes generadas [17].

Para realizar el análisis se calcularon las siguientes propiedades de centralidad, con el objetivo de establecer la influencia de los actores (el grado en que un autor tiene influencia en los recursos) [29-30]: centralidad de grado, centralidad de intermediación y centralidad de cercanía $[8,11]$. Además, para el análisis de datos bibliográficos se utilizó el software Bibexcel [31] y para la visualización de las redes se utilizaron Gephi [32] y Netdraw [33]. Para calcular las propiedades de la red se utilizó UCINET [34]. El uso de dos software diferentes para realizar la visualización se debe a la búsqueda de la mayor claridad visual en las redes construidas.

\section{ANÁLISIS DE RESULTADOS}

\section{Construcción de mapas del conocimiento de intenciones emprendedoras}

Inicialmente se muestra la evolución temporal de las publicaciones en el campo de las intenciones emprendedoras, constituidas por los 202 artículos resultantes de la ecuación de búsqueda utilizada en SCOPUS (ver Figura 1). Inicialmente hay un comportamiento constante caracterizado por pocas publicaciones en el tema de estudio; sin embargo, a partir de 2005 hay un comportamiento creciente en el número de publicaciones. Lo anterior muestra el interés progresivo de los investigadores en la temática de intenciones emprendedoras, la idoneidad y pertinencia de estas investigaciones como un aporte al entendimiento de la creación de empresas.

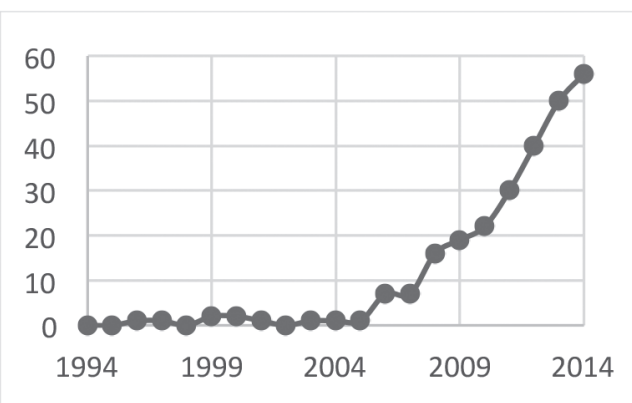

Figura 1. Evolución temporal de las publicaciones en el campo de las intenciones emprendedoras.

Respecto del área temática, en la Tabla 2 (elaborada a partir de los resultados de SCOPUS) se observa el número de artículos por área, observando cómo los estudios en intenciones emprendedoras se ubican principalmente en las áreas de Business, Management and Accounting, Economics (72,3\%), Econometrics and Finance $(34,2 \%)$ y Social Sciences $(29,7 \%)$. La sumatoria de los porcentajes no corresponde al $100 \%$, ya que una investigación puede estar categorizada en diversas áreas del conocimiento al mismo tiempo.

Además, se observa que las investigaciones alrededor de la intención emprendedora presentan un carácter multidisciplinar, combinando la teoría con la práctica e incrementado el uso de herramientas

Tabla 2. Número de artículos por tema de investigación.

\begin{tabular}{|c|c|c|}
\hline Área temática & $\begin{array}{l}\text { Número de } \\
\text { artículos }\end{array}$ & $\%$ \\
\hline $\begin{array}{c}\text { Business, Management and } \\
\text { Accounting }\end{array}$ & 146 & 72,3 \\
\hline $\begin{array}{c}\text { Economics, Econometrics and } \\
\text { Finance }\end{array}$ & 69 & 34,2 \\
\hline Social Sciences & 60 & 29,7 \\
\hline Psychology & 18 & 8,91 \\
\hline Decision Sciences & 6 & 2,97 \\
\hline Multidisciplinary & 5 & 2,48 \\
\hline Engineering & 5 & 2,48 \\
\hline Arts and humanities & 2 & 0,99 \\
\hline Neuroscience & 1 & 0,50 \\
\hline Enviromental sciences & 1 & 0,50 \\
\hline Mathematics & 1 & 0,50 \\
\hline Computer Science & 1 & 0,50 \\
\hline $\begin{array}{c}\text { Pharmacology, toxicology and } \\
\text { pharmaceutics }\end{array}$ & 1 & 0,50 \\
\hline $\begin{array}{c}\text { Biochemistry, Genetics and } \\
\text { molecular Biology }\end{array}$ & 1 & 0,50 \\
\hline
\end{tabular}


sistémicas en las investigaciones. Lo anterior es un comportamiento acorde con la satisfactoria evolución de un campo de estudio.

Para el análisis de la red visual de coautores (Figura 2, elaborada con el software Netdraw [33]), se consideraron los autores con mayor frecuencia para efectos de clara visualización; sin embargo, para efecto de cálculos se consideró toda la red. Se trabajó con 385 nodos y el top de los 10 autores con mayores centralidades es presentado en la Tabla 3. Liñan es el autor con mayor número de uniones con los demás autores y además es el que presenta la mayor cercanía a los demás nodos, controlando el flujo de información y jugando un papel de mayor intermediario entre los otros autores que no cuentan con contacto directo. Debido a estas características, Liñan ha publicado con diversos autores que guardan relación entre ellos y además están relacionados con temas multidisciplinares. Un caso interesante se presenta con los autores Toney y Pruett, quienes a pesar de estar directamente vinculados con un gran número de autores y de estar relativamente cerca de estos, no presentan papel de intermediarios entre otros autores, lo que podría denotar que sus investigaciones son en un campo del conocimiento específico y no gozan de multidisciplinaridad temática.

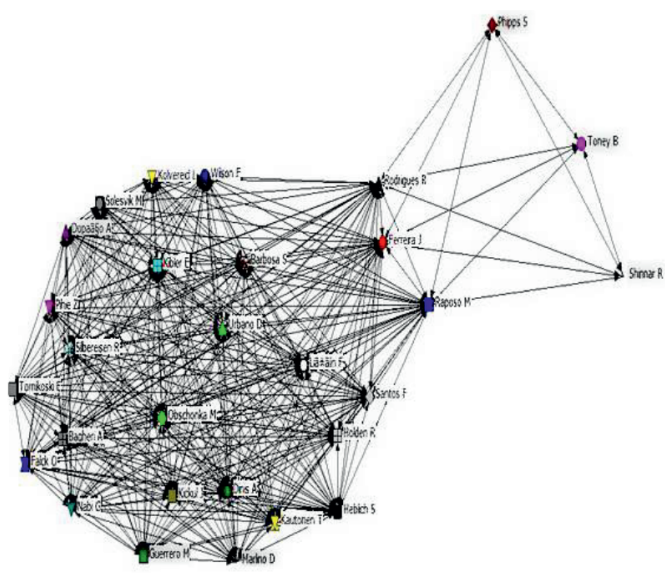

Figura 2. Red de autores.

Es importante recordar que el desempeño de un investigador está positivamente asociado con sus medidas de centralidad y la posición de un investigador dentro de la red de colaboración. Así, los investigadores conectados con el mayor número de autores y quienes presentan menos caminos cortos con cada uno de los otros autores presentan mejores desempeños en el campo de investigación al presentar el mayor prestigio y ser los que tienen mayores intermediarios en los flujos de información.

Tabla 3. Resumen de autores considerando propiedades de centralidad.

\begin{tabular}{|c|c|c|}
\hline $\begin{array}{c}\text { Centralidad de } \\
\text { grado }\end{array}$ & $\begin{array}{c}\text { Centralidad de } \\
\text { cercanía }\end{array}$ & $\begin{array}{c}\text { Centralidad de } \\
\text { intermediación }\end{array}$ \\
\hline Liñan & Liñan & Liñan \\
\hline Ferreira & Tornikoski & Tornikoski \\
\hline Raposo & Ferreira & Vangelderen \\
\hline Rodrigues & Kautonen & Kautonen \\
\hline Tornikoski & Raposo & Urbano \\
\hline Obschonka & Rodrigues & Ferreira \\
\hline Silbereisen & Obschonka & Obschonka \\
\hline Kautonen & Silbereisen & Silbereisen \\
\hline Toney & Toney & Raposo \\
\hline Pruett & Pruett & Rodrigues \\
\hline
\end{tabular}

Para la red de centros de investigación cada centro de investigación es un autor. La red resultante se presenta en la Figura 3, la que cuenta con 303 nodos y 622 vínculos. Allí se consideraron los centros de investigación con mayor frecuencia para efectos de clara visualización, sin embargo para efecto de cálculos se consideró toda la red. En la Tabla 4 se observan los resultados para los centros de investigación, considerando los diversos tipos de centralidad.

Los resultados obtenidos tanto para el grado de centralidad como para la centralidad de cercanía

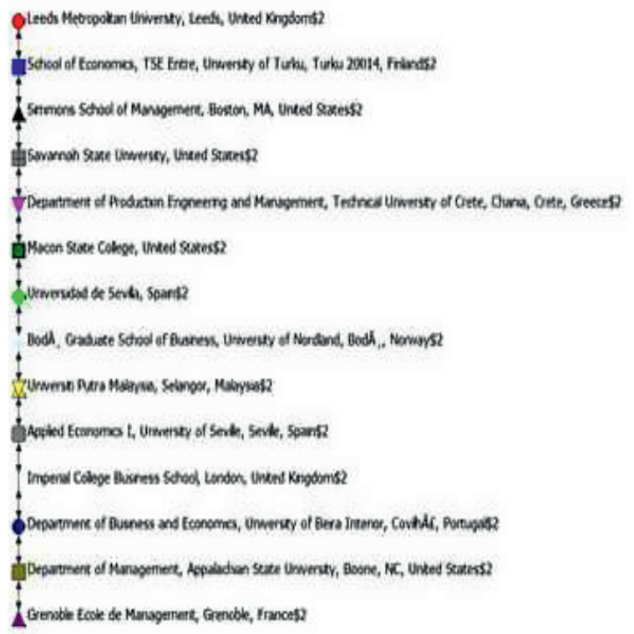

Figura 3. Redes de centros de investigación. Elaborada usando Netdraw [33]. 
son iguales, lo que indica que los mismos centros de investigación que dominan el flujo de información también tienen mayor influencia sobre otros, respecto del estudio de las intenciones emprendedoras. Lo anterior no implica que estos sean los centros que más investiguen respecto de la temática, sino que sus investigaciones gozan de gran reconocimiento y afectan en mayor proporción el trabajo de los otros centros de investigación.

Tabla 4. Resumen de centros de investigación considerando propiedades de centralidad.

\begin{tabular}{|c|c|c|}
\hline $\begin{array}{c}\text { Grado de } \\
\text { centralidad } \\
\text { (valor) }\end{array}$ & $\begin{array}{l}\text { Grado de } \\
\text { cercanía }\end{array}$ & $\begin{array}{c}\text { Grado de } \\
\text { intermediación }\end{array}$ \\
\hline $\begin{array}{c}\text { Melbourne } \\
\text { Business School, } \\
\text { Australia }\end{array}$ & $\begin{array}{l}\text { Melbourne } \\
\text { Business } \\
\text { School, } \\
\text { Australia }\end{array}$ & $\begin{array}{c}\text { Imperial College } \\
\text { Business School, } \\
\text { London }\end{array}$ \\
\hline $\begin{array}{c}\text { Department } \\
\text { of Managerial } \\
\text { Studies, } \\
\text { University of } \\
\text { Illinois } \\
\end{array}$ & $\begin{array}{c}\text { Department } \\
\text { of Managerial } \\
\text { Studies, } \\
\text { University of } \\
\text { Illinois } \\
\end{array}$ & $\begin{array}{c}\text { Simmons School } \\
\text { of Management, } \\
\text { Boston }\end{array}$ \\
\hline $\begin{array}{l}\text { Sabanci } \\
\text { University, } \\
\text { Faculty of } \\
\text { Management, } \\
\text { Istanbul, Turkey }\end{array}$ & $\begin{array}{c}\text { Sabanci } \\
\text { University, } \\
\text { Faculty of } \\
\text { Management, } \\
\text { Istanbul, } \\
\text { Turkey }\end{array}$ & $\begin{array}{l}\text { Bodø Graduate } \\
\text { School of } \\
\text { Business, } \\
\text { University of } \\
\text { Nordland }\end{array}$ \\
\hline $\begin{array}{l}\text { Indian Institute } \\
\text { of Management, } \\
\text { Indore, India }\end{array}$ & $\begin{array}{c}\text { Indian } \\
\text { Institute of } \\
\text { Management, } \\
\text { Indore, India }\end{array}$ & $\begin{array}{c}\text { Grenoble Ecole } \\
\text { de Management, } \\
\text { Grenoble }\end{array}$ \\
\hline $\begin{array}{c}\text { Radboud } \\
\text { University } \\
\text { Nijmegem, } \\
\text { Nijmegen, } \\
\text { Netherlands }\end{array}$ & $\begin{array}{l}\text { Radboud } \\
\text { University } \\
\text { Nijmegem, } \\
\text { Nijmegen, } \\
\text { Netherlands }\end{array}$ & $\begin{array}{c}\text { Sabanci } \\
\text { University, } \\
\text { Faculty of } \\
\text { Management, } \\
\text { Istanbul }\end{array}$ \\
\hline $\begin{array}{l}\text { Maastricht } \\
\text { University, } \\
\text { Maastricht, } \\
\text { Netherlands }\end{array}$ & $\begin{array}{l}\text { Maastricht } \\
\text { University, } \\
\text { Maastricht, } \\
\text { Netherlands }\end{array}$ & $\begin{array}{c}\text { University of } \\
\text { Iowa, United } \\
\text { States }\end{array}$ \\
\hline $\begin{array}{c}\text { University of } \\
\text { Wollongong, } \\
\text { College of } \\
\text { Business, Dubai }\end{array}$ & $\begin{array}{l}\text { University of } \\
\text { Wollongong, } \\
\text { College of } \\
\text { Business, } \\
\text { Dubai }\end{array}$ & $\begin{array}{c}\text { Syracuse } \\
\text { University, United } \\
\text { States }\end{array}$ \\
\hline $\begin{array}{c}\text { Massey } \\
\text { University, } \\
\text { Auckland } \\
\end{array}$ & $\begin{array}{c}\text { Massey } \\
\text { University, } \\
\text { Auckland } \\
\end{array}$ & $\begin{array}{l}\text { Maastricht } \\
\text { University, } \\
\text { Maastricht }\end{array}$ \\
\hline $\begin{array}{c}\text { Imperial College } \\
\text { Business School, } \\
\text { London }\end{array}$ & $\begin{array}{l}\text { Imperial } \\
\text { College } \\
\text { Business } \\
\text { School, } \\
\text { London }\end{array}$ & $\begin{array}{c}\text { School of } \\
\text { Economics and } \\
\text { Management, } \\
\text { Tongji University, } \\
\text { Shanghai }\end{array}$ \\
\hline $\begin{array}{l}\text { School of } \\
\text { Management, } \\
\text { Binghamton } \\
\text { University, } \\
\text { Binghamton }\end{array}$ & $\begin{array}{c}\text { School of } \\
\text { Management, } \\
\text { Binghamton } \\
\text { University, } \\
\text { Binghamton }\end{array}$ & $\begin{array}{l}\text { Rensselaer } \\
\text { Polytechnic } \\
\text { Institute }\end{array}$ \\
\hline
\end{tabular}

Por lo demás, respecto de la centralidad de intermediación el Imperial College Business School juega el papel de principal intermediario del flujo de información entre los diferentes centros de investigación y, además, respecto del grado de centralidad y al grado de cercanía, cuenta con gran prestigio e influencia respecto de los otros centros de investigación.

En las KCO network, cada palabra clave es un autor. La red resultante se presenta en la Figura 4, la que cuenta con 1631 vínculos y 689 nodos, en donde se consideran las palabras claves con mayor frecuencia para efectos de clara visualización y los cálculos sí involucran toda la red. En la Tabla 5 se observan las 9 palabras claves considerando los diversos tipos de centralidad.

Tabla 5. Resumen de palabras claves considerando propiedades de centralidad.

\begin{tabular}{|c|c|c|}
\hline $\begin{array}{c}\text { Grado de } \\
\text { centralidad }\end{array}$ & Grado de cercanía & $\begin{array}{c}\text { Grado de } \\
\text { intermediación }\end{array}$ \\
\hline $\begin{array}{c}\text { Entrepreneurial } \\
\text { intentions }\end{array}$ & Students & $\begin{array}{c}\text { Entrepreneurial } \\
\text { intentions }\end{array}$ \\
\hline Entrepreneurship & $\begin{array}{c}\text { Entrepreneurial } \\
\text { intentions }\end{array}$ & Entrepreneurship \\
\hline Entrepreneurialism & $\begin{array}{c}\text { Theory of planned } \\
\text { Behavior }\end{array}$ & Students \\
\hline $\begin{array}{c}\text { Entrepreneurship } \\
\text { education }\end{array}$ & Entrepreneurship & $\begin{array}{c}\text { Theory of planned } \\
\text { Behavior }\end{array}$ \\
\hline Education & Self-efficacy & Entrepreneurialism \\
\hline Attitudes & Spain & Entrepreneurship \\
\hline Gender & $\begin{array}{c}\text { Entrepreneurialism } \\
\text { Entrepreneurship } \\
\text { education }\end{array}$ \\
\hline Students & $\begin{array}{c}\text { Entrepreneurship } \\
\text { education }\end{array}$ & Education \\
\hline China & Gender & Self-efficacy \\
\hline $\begin{array}{c}\text { Developing } \\
\text { countries }\end{array}$ & $\begin{array}{c}\text { Structural Equation } \\
\text { Modelling }\end{array}$ & Attitudes \\
\hline
\end{tabular}

La red de temas de investigación permite desde el micronivel identificar los principales temas y la evolución de un campo de conocimiento, con base en la coocurrencia de palabras clave, ya que estas representan las ideas centrales de un trabajo de investigación. Se destaca que las principales disciplinas que contribuyen en la información recuperada de la base de datos son: Business, Management and Accounting, Economics, Econometrics and Finance, Social Sciences, Psychology y Decision Sciences. Esto es acorde con las tendencias investigativas de los últimos años en el área de intención emprendedora, la que se ha 
enfocado en aspectos como la viabilidad, riesgo, creación y contexto económico para la creación de empresas desde la perspectiva del individuo. Lo anterior permite analizar la forma en que diversos factores afectan la toma de decisiones al momento de crear empresas y cómo los diversos factores psicológicos asociados a cada individuo pueden inhibir o catalizar el paso de la intención a la acción de emprender.

Al indagar acerca de las temáticas de estudio específicas, en la Tabla 5 se observan las 10 palabras claves más frecuentes en los artículos relacionados con intención emprendedora, estas son: Entrepreneurial intentions, Entrepreneurship, Students, Entrepreneurialism, Theory of planned Behavior, Education, Entrepreneurship Education, Gender, Self-efficacy y Attitudes. Estas palabras ofrecen orientación respecto de las teorías y a los constructos que han sido utilizados para estudiar el tema de las intenciones emprendedoras. Igualmente se destacan aspectos importantes respecto de la población objetivo a la que han sido dirigidos los estudios, permitiendo identificar que los principales grupos son: estudiantes y grupos por género, específicamente mujeres.

Otras palabras claves que se observan en la red de temas relacionan el "empleo" con las "intenciones emprendedoras" y con el "desarrollo de un país". Lo anterior podría dar indicios de la orientación de las investigaciones al tratar el tema de la intención de emprender como resultado de la motivación de los individuos por autoemplearse, situación que podría favorecer el crecimiento económico del país mediante la generación de nuevas empresas. Además, palabras como social capital y social entrepreneurship, ligadas al concepto de "normas subjetivas", muestran cómo el emprendedor puede optar por tipos de emprendimiento que buscan satisfacer necesidades sociales en los que las normas sociales tienen gran importancia al primar aspectos diferentes al lucro en sus intenciones emprendedoras.

En la Figura 4 (elaborada usando Gephi [32]) se observa que respecto del grado de centralidad la palabra con mayor cantidad de vínculos con las otras fue Entrepreneurial intentions, situación que era esperada si se considera que la primera estaba asociada a la búsqueda. Por otro lado, las palabras que continúan en grado de centralidad están asociadas a

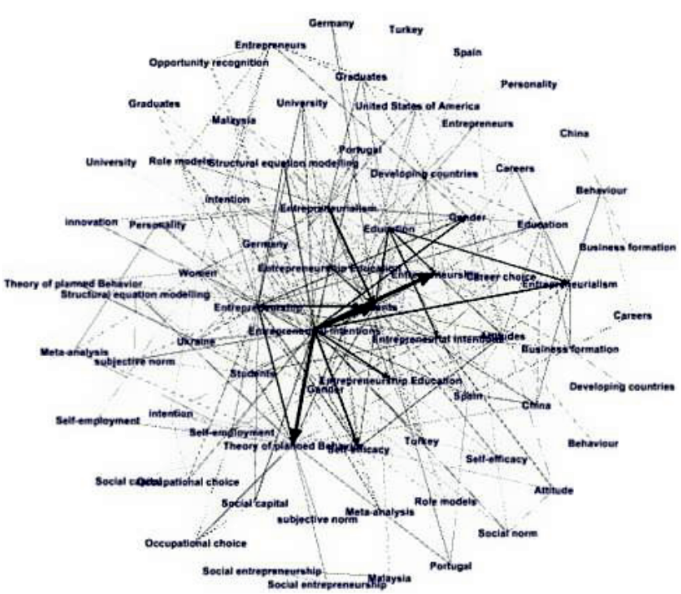

Figura 4. Red de temas de investigación.

los temas dentro de los cuales se encuentra inmersa el área de interés. Es importante resaltar que los estudios relativos a "educación emprendedora", "intenciones emprendedoras en estudiantes" y "género", son muy comunes. Esto evidencia un interés en segmentar la población de interés con el fin de tener caracterizaciones particulares que permitan identificar los aspectos que se deben incentivar, dependiendo el tipo de individuo que se esté analizando. Respecto del grado de intermediación, hay similitudes en cuanto al análisis de la centralidad de grado. Sin embargo, es importante observar que las palabras "Teoría del Comportamiento Planeado (TCP)", "autoeficacia" y "actitudes" indican los conceptos respecto de los que subyace la temática de las intenciones emprendedoras, ya que dan origen a los constructos más comunes de análisis en los modelos de intención emprendedora, como es el caso del modelo de Ajzen [35]. Este modelo hace referencia a la Teoría del Comportamiento Planeado (TCP), y de él se han derivado otros modelos de estudio de la intención emprendedora con los que se tienen diversos constructos en común, como: las actitudes, normas subjetivas, control de comportamiento percibido, comportamiento emprendedor, entre otras. Además, se ha buscado verificar si el modelo de la TCP, y sus variantes, son adecuados para detectar las intenciones emprendedoras [36]. Lo anterior es un indicativo de la frecuencia con que estos conceptos se encuentran en la mayoría de los estudios concernientes a intenciones emprendedoras. Entre los temas de estudio potenciales se encuentra el análisis de la pertenencia a redes como factor de impulso a la intención emprendedora, ya que se ha 
convertido en una de las variables más representativas en las investigaciones enfocadas en el proceso de creación de empresas [37].

Finalmente, respecto del grado de cercanía, se observan rasgos comunes en cuanto a las propiedades anteriores, pero cabe destacar que las palabras Structural Equation Modelling y student indican que estos conceptos se encuentran muy cercanos a los otros por medio de muchas conexiones cortas. Es decir, se encuentran muy cerca de muchos elementos de la red, siendo muy centrales e indicando la gran cantidad de veces que los estudios involucran estudiantes de forma directa o indirecta como grupo de estudio, y el hecho de que los Modelos de Ecuaciones Estructurales, Structural Equation Modelling (SEM) están siendo utilizados como herramienta estadística para la interpretación y análisis de los datos obtenidos en el trabajo de campo. Los Modelos de Ecuaciones Estructurales hacen referencia a modelos estadísticos multivariantes que permiten estimar el efecto y las relaciones entre múltiples variables y constructos. Estos son menos restrictivos que los modelos de regresión, ya que permiten la inclusión de errores de medida, tanto en las variables criterio (dependientes) como en las variables predictoras (independientes) [38]. Como se observa en el mapeo de conocimiento de intenciones emprendedoras, los Modelos de Ecuaciones Estructurales son ampliamente utilizados debido a que permiten proponer el tipo y dirección de las relaciones que se espera encontrar entre los diferentes constructos que componen los modelos de intención emprendedora, permitiendo estimar la validez de dichas relaciones teóricas de una forma práctica.

\section{CONCLUSIONES}

La intención emprendedora se ha convertido con el paso del tiempo en un campo de investigación importante, que requiere un análisis sistemático de su estructura de conocimiento. Por tanto, se adoptó una metodología replicable en otros campos investigativos que ha permitido, por un lado, encontrar los autores más productivos en términos de desempeño y por otro, identificar las principales temáticas y enfoques abordados en el tema de intenciones emprendedoras realizando consideraciones temporales. Esta metodología combina el análisis de palabras con las redes de colaboración, permitiendo mapear el conocimiento, ya que relaciona los artículos entre ellos, mostrando el grado de relación entre las investigaciones. Igualmente, las redes de coautoría implican un estrecho vínculo social; ya que involucra una relación temporal y una actividad común. Asimismo, el uso de SCOPUS ha permitido que se obtengan documentos que sean representativos de la temática al realizar consideraciones de amplitud, calidad (indexación) y multidisciplinariedad.

El estudio de las intenciones emprendedoras es principalmente abordado por áreas temáticas como la administración, la economía y las ciencias sociales; ya que en estas se localiza el principal interés al considerar el impacto de los emprendimientos resultantes del proceso de intención. De forma similar se explican los estudios en el campo de la psicología, ya que los principales constructos en los que se sustentan los modelos de intención emprendedora se originan en esta área del conocimiento. Sin embargo, es importante resaltar algunos estudios que abordan temáticas como las ciencias de la salud, las artes y las ciencias ambientales, en los que se observa el amplio espectro de posibilidades de estudio de la intención emprendedora; así como su potencial para aportar a solucionar problemas desde otros ámbitos del saber.

Por otro lado, los autores se centran en los temas tradicionales de estudio, lo que hace que se encuentre gran conexión entre ellos, pero desemboca en poca diversidad y un bajo interés en nuevos temas. Esto se observa claramente en la red de autores donde se muestra a quienes tratan temas poco estudiados en la periferia e inclusive desconectados de la red principal (elemento mayor). Otra forma de ver este fenómeno es con la segmentación de estudios. Es común encontrar estudios realizados a estudiantes que se encuentran próximos a salir al entorno laboral, o estudios regionales para validar los modelos en poblaciones particulares y contextos locales.

Frente a las posibilidades de trabajos futuros, es importante resaltar que con base en el análisis de las conexiones de las palabras claves, se encuentran pocos estudios que propendan por aplicar nuevas metodologías. Por ejemplo, solo dos documentos en sus palabras claves o abstract desarrollaron el concepto de análisis longitudinal. Por tanto, las temáticas se centran en áreas y metodologías tradicionales y se destaca la poca diversidad en 
nuevos temas. Esto se observa claramente en la red de temas de investigación, en donde se muestra a los temas poco estudiados en la periferia, mientras que la gran densidad de la red se asocia al estudio de pocas áreas del conocimiento.

Por lo anterior, se recomienda ampliar el número y objeto de las investigaciones, lo que permitirá aumentar la participación de los autores y sus interacciones con otros. Lo anterior solo será posible si se amplía el número de campos a investigar y se incentiva la interdisciplinariedad en el campo de las intenciones emprendedoras, garantizando la diversidad temática y el estudio o generación de nuevos campos de estudio. Es claro que la Teoría del Comportamiento Planeado ha sido la base conceptual sobre la cual se han construido la mayoría de los estudios; igualmente the Structural Equation Modelling ha sido la metodología que más se ha utilizado para el análisis e interpretación de resultados. Por tanto, la ampliación más allá de estas teorías y técnicas favorecería la diversidad temática y la generación de nuevos campos de estudio en el área de las intenciones emprendedoras. Por lo anterior, se sugiere la incorporación de visiones no lineales apoyadas en herramientas sistémicas que ayuden a brindar un mejor acercamiento al entendimiento del dinamismo y las relaciones de causalidad presentes en las variables que componen los modelos de intención emprendedora.

\section{REFERENCIAS}

[1] M. Gupta and S. Bhattacharya. "A Bibliometric Approach towards Mapping the Dynamics of Science and Technology". DESIDOC. Vol. $24 \mathrm{~N}^{\mathrm{o}}$ 1, pp. 3-8. 2004.

[2] A. Rorissa and X. Yuan. "Visualizing and mapping the intellectual structure of information retrieval". Information Processing \& Management. Vol. $48 \mathrm{~N}^{\circ} 1$, pp. 120-135. 2012. DOI: 10.1016/j.ipm.2011.03.004

[3] B. Quesada, D. Minguillo, Z. ChinchichaRodríguez y F. de Moya-Anegón. "Estructura de la colaboración científica española en Biblioteconomía y Documentación" (Scopus 1999-2007). Rev. Interam. Bibliot. Vol. 33 $\mathrm{N}^{\circ} 1.2010$.

[4] N.F. Krueger, M. D. Reilly and A. L. Carsrud. "Competing models of entrepreneurial intentions". Journal of business venturing. Vol. $15 \mathrm{~N}^{\circ}$ 5, pp. 411-432. 2002.

[5] M. Pina. "Entrepreneurship As Decision Making: Rational, Intuitive And Improvisational Approaches". Journal of Enterprising Culture (JEC). Vol. $15 \mathrm{~N}^{\mathrm{o}} 1$, pp. 1-20. 2007.

[6] F. Requena. "El concepto de red social". Revista Española de Investigaciones Sociológicas. Vol. 48, pp. 137-152. 1989

[7] C. Hirschi. "Introduction: Applications of Social Network Analysis". Procedia - Social and Behavioral Sciences. Vol. 4, pp. 2-3. 2010. DOI:10.1016/j.sbspro.2010.07.477

[8] X. Liu, J. Bollen, M.L. Nelson y H. Van de Sompel. "Co-authorship networks in the digital library research community". Information Processing \& Management. Vol. $41 \mathrm{~N}^{\circ}$ 6, pp. 1462-1480. 2005. DOI:10.1016/j.ipm.2005.03.012

[9] H.N. Su y P. C. Lee. "Framing the structure of global open innovation research". Journal of Informetrics, Vol. $6 \mathrm{~N}^{\mathrm{o}} 2$, pp. 202-216. 2012. DOI:10.1016/j.joi.2011.12.001.

[10] M. Tsevetovat y A. Kouznetsov. "Social Network Analysis for Startups: Finding connections on the social web". O'RRELLY, pp. 1-192. 2011.

[11] S. Wasserman and K. Faust. "Social Network Analysis: Methods and Applications". Cambridge University Press. Cambridge, Reino Unido, pp. 1-9. 1994. ISBN: 0-521-38269-6.

[12] M. Newman. "Networks: An Introduction". Oxford University Press. Oxford, Inglaterra. 2010. ISBN: 0-19-920665-1.

[13] M. Martino and M Pósfai. "Barabási. Network Science". Chapter one, pp. 1-10. 2012. URL: http://barabasilab.com/networksciencebook. Fecha de Consulta: 19 de noviembre de 2014.

[14] M. Cobo, A. G. López-Herrera, E. HerreraViedma and F. Herrera. "An approach for detecting, quantifying, and visualizing the evolution of a research field: A practical application to the Fuzzy Sets Theory field". Journal of Informetrics. Vol. $5 \mathrm{~N}^{\mathrm{o}} 1$, pp. 146166. 2012. DOI:10.1016/j.joi.2010.10.002

[15] E. Peláez, L. Cadavid y G. Awad. "Propuesta Metodológica para Realizar una Vigilancia Tecnológica Apoyada en un Análisis Topológico de Redes de Autores". XIV congreso latino - iberoaméricano de gestión 
tecnológica - ALTEC. Innovación para el crecimiento sostenible en el marco del bicentenario. Lima, Perú. 2010.

[16] L. Cadavid, G. Awad y J. Cardona. "Análisis bibliométrico del campo Modelado de Difusión Innovaciones”. Estudios Gerenciales. Vol. 31 $\mathrm{N}^{\circ}$ 135, pp. 213-236. 2012.

[17] H. Su and P. Lee. "Mapping knowledge structure by keyword co-occurrence: A first look at journal papers in Technology Foresight". Scientometrics. Vol. $85 \mathrm{~N}^{\mathrm{o}} 1$, pp. 65-79. 2010.

[18] M. Postigo, J. Díaz y R. Hernández. "Revisión de la literatura en fracaso empresarial: aproximación bibliométrica". In Estableciendo puentes en una economía global. Escuela Superior de Gestión Comercial y Marketing, ESIC, pp. 102. 2008.

[19] M. P. Viana, D. R. Amancio, F. Da and L. Costa. "On time-varying collaboration networks". Journal of Informetrics. Vol. 7 $\mathrm{N}^{\mathrm{o}}$ 2, pp. 371-378. 2013. DOI:10.1016/j. joi.2012.12.005

[20] S. Fortunato. "Community detection in graphs". Physics Reports, Vol. 486 No 3-5, pp. 75-174. 2010. URL: http://www. sciencedirect.com/science/article/pii/S03701 57309002841. Fecha de Consulta: 10 de noviembre de 2014.

[21] D.H. Sonnenwald. "Scientific collaboration". Ann. Rev. Info. Sci. Tech. Vol. 41, pp. 643-681. 2007. DOI: 10.1002/aris.2007.1440410121

[22] C.S. Wagner and L. Leydesdorff. "Network structure, self-organization, and the growth of international collaboration in science". Research Policy. Vol. 34 N$^{\circ}$ 10, pp. 1608-1618, 2005. DOI:10.1016/j.respol.2005.08.002

[23] K. Abbasi, S. Chung and L. Hossain. "Egocentric analysis of co-authorship network structure, position and performance". Information Processing \& Management. Vol. $48 \mathrm{~N}^{\circ}$ 4, pp. 671-679, 2012. DOI:10.1016/j.ipm.2011.09.001

[24] M. Girvan and M.E. Newman. "Community structure in social and biological networks". Proceedings of the National Academy of Sciences of the United States of America. Vol. $99 \mathrm{~N}^{\mathrm{o}} 12$, pp. 7821-7826. 2002. DOI:10.1073/pnas.122653799.

[25] J. Mitre y L. Navarro-Moldes. "P2P Protocol to Scientific Collaboration Networks, an Approach", pp. 1-9. 2004.
[26] Y. Rodríguez. “Trilogía para la visión científica: las publicaciones científicas, las bases de datos y la bibliometría". Biblios. Vol. $31 \mathrm{~N}^{\circ}$ 3, pp. 1-9. 2008.

[27] J. De Granda-Orive, A. Alonso-Arroyo and F. Roig-Vázquez. "Which Data Base Should we Use for our Literature Analysis? Web of Science versus SCOPUS". Archivos de Bronconeumología. Vol. 47 No 4, pp. 213. 2011. DOI: 10.1016/S1579-2129(11)70049-0.

[28] J. Burnham. "Scopus database: a review". Biomedical digital libraries. Vol. $3 \mathrm{~N}^{\mathrm{o}} 1$. 2006. DOI: $10.1186 / 1742-5581-3-1$.

[29] D.J. Brass and M.E. Burkhardt "Centrality and Power in Organizations”. In N. Nohria, \& R. G. Eccles (Eds.), Networks and Organizations: Structure, Form, and Action. Cambridge: President and Fellows of Harvard College. 1992.

[30] L. Freeman. "Centrality in social networks: Conceptual clarification". Social networks. Vol. $1 \mathrm{~N}^{\circ}$ 3, pp. 215-239. 1979.

[31] O. Persson, R. Danell, J Wiborg. "How to use Bibexcel for various types of bibliometric analysis". In Celebrating scholarly communication studies: A Festschrift for Olle Persson at his 60th Birthday, ed. F. Åström, R. Danell, B. Larsen, J. Schneider, pp. 9-24. Leuven, Belgium: International Society for Scientometrics and Informetrics. 2009.

[32] M. Bastian, S. Heymann and M. Jacomy. "Gephi: an open source software for exploring and manipulating networks". International AAAI Conference on Weblogs and Social Media. 2009.

[33] S. P. Borgatti. "NetDraw" [computer software]. Harvard, MA. Analytic Technologies. 2002.

[34] S.P. Borgatti, M.G. Everett and L.C. Freeman. "Ucinet for Windows: Software for Social Network Analysis". Analytic Technologies. Harvard, MA, USA. 2002.

[35] I. Ajzen. "The theory of planned behavior". Organizational Behavior and Human Decision Processes. Vol. 50 No 2, pp. 179-211. 1991.

[36] A.Valencia, L. Cadavid, D.C. Ríos, y G. Awad. "Factores que inciden en las intenciones emprendedoras de los estudiantes". Revista Venezolana de Gerencia. Vol. 17 N 57 , pp. 132-148. 2012. 
[37] A. Valencia, J. Bermudez, y A. Jaramillo. "El papel de la pertenencia a redes en el proceso de creación de empresas". Revista Espacios. Vol. $34 \mathrm{~N}^{\circ}$ 11, pp. 7-15. 2013.
[38] M. Ruiz, A. Pardo y R. San Martin. "Modelos de Ecuaciones Estructurales". Papeles del psicólogo. Vol. $31 \mathrm{~N}^{\circ} 1$, pp. 34-45. 2010. 